\title{
ABSOLUTELY AREA MINIMIZING SINGULAR CONES OF ARBITRARY CODIMENSION
}

\author{
BY
}

\author{
DAVID BINDSCHADLER
}

\begin{abstract}
The examples of area minimizing singular cones of codimension one discovered by Bombieri, DeGiorgi and Guisti are generalized to arbitrary codimension, thus filling a dimensional gap. Previously the only nontrivial examples of singular area minimizing integral currents of codimension other than one were obtained from holomorphic varieties and hence of even codimension. Specifically, let $S$ be the $N$-fold Cartesian product of $p$-dimensional spheres and $C$ be the cone over $S$. We prove that for $p$ sufficiently large, $C$ is absolutely area minimizing. It follows from the technique used that $C$ restricted to the ball of radius $N^{1 / 2}$ is the unique solution to the oriented Plateau problem with boundary $S$.
\end{abstract}

1. Introduction. In 1960 Federer and Fleming introduced integral currents to prove existence of solutions to the oriented Plateau problem [FF]. Since that time much effort has been aimed at understanding the structure of these solutions. An important structural question is that of interior regularity. A major obstacle to the study of this question has been the lack of nontrivial examples where regularity fails. In [F1] Federer provided the first nontrivial examples of singular solutions by showing that holomorphic varieties are absolutely area minimizing. Subsequently, Federer proved that there exist tangent cones at every interior point of a solution and that such cones are themselves area minimizing [F2, 5.4.3]. Thus the search for types of singularities is reduced to a search for area minimizing cones. Bombieri, DeGiorgi, and Guisti provided the first nontrivial example of a singular solution to the oriented Plateau problem that did not arise from a holomorphic variety by showing that the cone over $S^{p} \times S^{p} \subset \mathbf{R}^{2(p+1)}$ is area minimizing whenever $p \geqslant 3$ [BGG]. Lawson [L] and Simoes [S] exhibited many more codimension one area minimizing cones of various topological type. Here we give examples of area minimizing oriented cones of any preassigned codimension greater than one.

Let $p$ and $N$ be positive integers with $N \geqslant 3$,

$$
U=\mathbf{R}^{N} \cap\left\{x: x_{i}>0, i=1, \ldots, N\right\},
$$

Received by the editors April 28, 1977.

AMS (MOS) subject classifications (1970). Primary 49F22, 49F10; Secondary 49F20, 58 A25.

Key words and phrases. Plateau problem, integral current, interior regularity, area minimizing cones.

- American Mathematical Society 1978 
and $\pi:\left(\mathbf{R}^{p+1}\right)^{N} \rightarrow$ Clos $U$ be defined by

$$
\pi\left(y_{1}, \ldots, y_{N}\right)=\left(\left|y_{1}\right|, \ldots,\left|y_{N}\right|\right),
$$

where $y_{i} \in \mathbf{R}^{p+1}$ for $i=1, \ldots, N$. If $L$ denotes the ray in $\mathbf{R}^{N}$ extending from the origin through $(1, \ldots, 1)$, then $C=\pi^{-1}(L)$ is the cone through $\pi^{-1}\{(1, \ldots, 1)\}=\left(S^{p}\right)^{N}$. Denote by $\Psi$ the integrand of degree 1 which assigns to a tangent vector at $u \in U$ its length times the Hausdorff $N p$ dimensional measure of $\pi^{-1}\{u\}$. Generalizing the function used in [L] we construct in $\$ 3$ a closed 1 -form $\varphi$ which proves that $L$ is $\Psi$ minimizing provided $N=3$ and $p>3$, or $N>3$ and $p>\left(2(N-1)^{1 / 2}-1\right) /(N-2(N$ $-1)^{1 / 2}$ ). Using the technique of $[\mathbf{F 3}, 6.3]$, in $\S 4 \mathrm{we}$ lift $\varphi$ to a flat cochain in $\left(\mathrm{R}^{p+1}\right)^{N}$ which is used to show that $C$ minimizes area with respect to both real and integer coefficients provided also $p>N-2$. In particular it follows that $C L B(0,1)^{N}$ is the unique area minimizing integral current with boundary $\left(S^{p}\right)^{N}$.

The bound on $p$ given here is better than the one obtained in $[B]$ and announced in Notices Amer. Math. Soc. (24 (1977), A-11, Abstract \#77T-B8). It implies that $C$ minimizes area if $N=3$ and $p>3, N=4$ and $p>5$, or $N>5$ and $p>N-1$.

I would like to express my gratitude to John E. Brothers for suggesting this problem and for his many helpful comments.

2. Notation. The standard coordinate functions for $\mathbf{R}^{N}$ will be denoted by $X^{i}, i=1, \ldots, N$. The $N$-dimensional spherical coordinate system for $U$ is the function

$$
\left(\rho, \theta^{1}, \ldots, \theta^{N-1}\right): U \rightarrow \mathbf{R}^{+} \times(0, \pi / 2)^{N-1}
$$

such that

$$
\begin{aligned}
& X^{1}=\rho \cos \theta^{1}, \quad X^{N}=\rho \prod_{j=1}^{N-1} \sin \theta^{j}, \\
& X^{i}=\rho \cos \theta^{i} \prod_{j=1}^{i-1} \sin \theta^{j}, \quad i=2, \ldots, N-1 .
\end{aligned}
$$

We will also use $\theta^{i}$ for the $i$ th coordinate function on the cube $(0, \pi / 2)^{N-1} \subset$ $\mathbf{R}^{N-1}$.

Let $x_{0}=\left(N^{-1 / 2}, \ldots, N^{-1 / 2}\right) \in U$ and $\theta_{0}^{i}=\theta^{i}\left(x_{0}\right), i=1, \ldots, N-1$. Then

$$
L=\left\{x \in U: \theta^{i}(x)=\theta_{0}^{i}, i=1, \ldots, N-1\right\} .
$$

For $1<j \leqslant k \leqslant N-1$, we use the notation

$$
S(j, k)=\prod_{i=j}^{k} \sin \theta^{i}, \quad C(j, k)=\prod_{i=j}^{k} \cos \theta^{i} .
$$


We will also use the conventions that if $j<k$, then $S(k, j)=C(k, j)=1$ and if $k>N-1$ or $k<1, \sin \theta^{k}=\cos \theta^{k}=1$.

For each positive integer $p$ define $V_{p}: U \rightarrow \mathbf{R}$ by

$$
V_{p}(u)=\mathcal{T}^{N p}\left(\pi^{-1}\{u\}\right) .
$$

Then

$$
\begin{aligned}
V_{p} & =(p+1)^{N} \alpha(p+1)^{N} \prod_{i=1}^{N}\left(X^{i}\right)^{p} \\
& =(p+1)^{N} \alpha(p+1)^{N} \rho^{N p} C(1, N-1)^{p}\left(\prod_{i=1}^{N-1} \sin ^{N-i} \theta^{i}\right)^{p},
\end{aligned}
$$

where $\alpha(p+1)$ denotes the volume of the unit $(p+1)$-ball.

Explanations of the remainder of the notation which we use can be found in [F2], which contains an index of notation on pp. 670-671.

\section{Basic computations.}

3.1. Let $j$ and $k$ be integers such that $1 \leqslant j<k<N-1$. Then

$$
\begin{gathered}
(N-k) \cot \theta_{0}^{k}-\tan \theta_{0}^{k}=0 . \\
S(j+1, k)^{2} C(1, j-1)^{2} C(j+1, k)^{2}\left((N-j) \cos ^{2} \theta^{j}-\sin ^{2} \theta^{j}\right)^{2} \\
=(N-j)^{2} S(j+1, k)^{2} C(1, k)^{2}+S(j, k)^{2} C(1, j-1)^{2} C(j+1, k)^{2} \\
-(N-j+1)^{2} S(j, k)^{2} C(1, k)^{2} . \\
\sum_{i=1}^{k}(N-i)^{2} S(i+1, k)^{2}-\sum_{i=1}^{k}(N-i+1)^{2} S(i, k)^{2} \\
=(N-k)^{2}-N^{2} S(1, k)^{2} .
\end{gathered}
$$

Proof. The proofs of statements (2) and (3) are straightforward. For the proof of (1) one uses induction on $k$ together with the observations

$$
\cos \theta_{0}^{1}=X^{1}\left(x_{0}\right)=N^{-1 / 2}, \quad \cos \theta_{0}^{k} \prod_{i=1}^{k-1} \sin \theta_{0}^{i}=N^{-1 / 2} .
$$

3.2. Let $A \neq 0, B, C$, and $D$ be real numbers and $H:[0, \pi / 2] \rightarrow \mathbf{R}$ be given by

$$
H(\theta)=A \cos ^{2} \theta \sin ^{2} \theta+B \sin ^{2} \theta+C \cos ^{2} \theta+D .
$$

If $A<0,|(C-B) / A|<1$ and $\cos 2 \theta_{1}=(C-B) / A$, then $H$ attains its minimum at $\theta_{1}$ and

$$
H\left(\theta_{1}\right)=(4 A)^{-1}\left((A+B+C)^{2}-4 B C\right)+D .
$$

Otherwise, $H$ attains its minimum at 0 or $\pi / 2$. 
Proof. The result can be established by elementary calculus.

3.3. If $\gamma_{i}>0, i=1, \ldots, k+1, k<N-2$, then the minimum value of the function $G:(0, \pi / 2)^{k} \rightarrow \mathbf{R}$, defined by

$$
G=\sum_{i=1}^{k} \gamma_{i} S(1, i-1)^{-2} \cos ^{-2} \theta^{i}+\gamma_{k+1} S(1, k)^{-2},
$$

is $\left(\sum_{i=1}^{k+1} \gamma_{i}^{1 / 2}\right)^{2}$.

Proof. It is easy to see that the function

$$
C_{0}+C_{1} \cos ^{-2} \theta+C_{2} \sin ^{-2} \theta, \quad 0<\theta<\pi / 2,
$$

has a minimum value of $C_{0}+\left(C_{1}^{1 / 2}+C_{2}^{1 / 2}\right)^{2}$ whenever $C_{i}>0, i=0,1,2$. The result then follows by minimizing $G$ with respect to each coordinate function starting with $\theta^{k}$.

3.4. THEOREM. If

(I) $N=3$ and $p>3$, or

(II) $N>3$ and

$$
p>\left(2(N-1)^{1 / 2}-1\right) /\left(N-2(N-1)^{1 / 2}\right),
$$

then there exists $\varphi \in \mathcal{E}^{1}(U)$ such that

(1) $d \varphi=0$,

(2) $\varphi\left|L=V_{p} d \rho\right| L$,

(3) $|\varphi|(x)<V_{p}(x)$ for all $x \in U$, with equality if and only if $x \in L$.

Proof. Define $h:[0, \pi / 2]^{N-1} \rightarrow \mathbf{R}$ and $g: U \rightarrow \mathbf{R}$ by

$$
h=C(1, N-1) \prod_{i=1}^{N-1} \sin ^{N-i} \theta^{i}, \quad g(x)=h\left(\theta^{1}(x), \ldots, \theta^{N-1}(x)\right) .
$$

Let $m_{0}=h\left(\theta_{0}^{1}, \ldots, \theta_{0}^{N-1}\right)^{-1}$. We will show that if (I) or (II) holds, then there exists a $\beta$ such that

$$
f=(N p+1)^{-1} \rho^{N p+1}\left(m_{0} g\right)^{\beta}
$$

satisfies

$$
\left(\frac{\partial f}{\partial \rho}\right)^{2}+\sum_{i=1}^{N-1} \rho^{-2} S(1, i-1)^{-2}\left(\frac{\partial f}{\partial \theta^{i}}\right)^{2}<m_{0}^{2 p} \rho^{2 N p} g^{2 p}
$$

with equality only on $L$. The theorem then follows with

$$
\varphi=(p+1)^{N} \alpha(p+1)^{N} m_{0}^{-p} d f .
$$

From

$$
\left(\frac{\partial h}{\partial \theta^{i}}\right)=\left((N-i) \cot \theta^{i}-\tan \theta^{i}\right) h
$$

one deduces 


$$
\left(\frac{\partial f}{\partial \theta^{i}}\right)=\beta(N p+1)^{-1} m_{0}^{\beta} \rho^{N p+1} g^{\beta}\left((N-i) \cot \theta^{i}-\tan \theta^{i}\right) .
$$

Then ( $\left.3^{\prime}\right)$ becomes

$$
\begin{aligned}
m_{0}^{2 p} \rho^{2 N p} g^{2 p} \geqslant & \rho^{2 N p} m_{0}^{2 \beta} g^{2 \beta} \\
+ & \sum_{i=1}^{N-1} S(1, i-1)^{-2} \beta^{2}(N p+1)^{-2} m_{0}^{2 \beta} \rho^{2 N p} g^{2 \beta} \\
& \cdot\left((N-i) \cot \theta^{i}-\tan \theta^{i}\right)^{2}
\end{aligned}
$$

or, equivalently,

$$
\begin{aligned}
0< & 1-\beta^{2}(N p+1)^{-2} m_{0}^{2 \beta-2 p} h^{2 \beta-2 p} \\
& \cdot\left[((N p+1) / \beta)^{2}+\sum_{i=1}^{N-1} S(1, i-1)^{-2}\left((N-i) \cot \theta^{i}-\tan \theta^{i}\right)^{2}\right] .
\end{aligned}
$$

Denote the right-hand side of the inequality by $F$ and note that $F\left(\theta_{0}^{1}, \ldots, \theta_{0}^{N-1}\right)=0$.

We will now assume $\beta>p+1$. Then

$$
h^{\beta-p} C(1, N-1)^{-1} S(1, N-1)^{-1}\left(\theta^{1}, \ldots, \theta^{N-1}\right) \rightarrow 0
$$

as any $\theta^{i}$ tends to 0 or $\pi / 2$. Hence $F\left(\theta^{1}, \ldots, \theta^{N-1}\right) \rightarrow 1$ as any $\theta^{i}$ tends to 0 or $\pi / 2$, so that it will suffice to find $\beta$ such that $\left(\theta_{0}^{1}, \ldots, \theta_{0}^{N-1}\right)$ is the only critical point of $F$ in $(0, \pi / 2)^{N-1}$.

Using (4) we obtain

$$
\begin{aligned}
& \partial F / \partial \theta^{N-1}=-2 \beta^{2}(N p+1)^{-2} m_{0}^{2 \beta-2 p} h^{2 \beta-2 p}\left(\cot \theta^{N-1}-\tan \theta^{N-1}\right) \\
& \cdot S(1, N-1)^{-2} C(1, N-1)^{-2} \\
& \cdot\left[(\beta-p) \beta^{-2}(N p+1)^{2} C(1, N-1)^{2} S(1, N-1)^{2}\right. \\
&-C(1, N-2)^{2}+\sum_{i=1}^{N-1}(\beta-p) S(i+1, N-1)^{2} C(1, i-1)^{2} \\
&\left.\cdot C(i+1, N-1)^{2}\left((N-i) \cos ^{2} \theta^{i}-\sin ^{2} \theta^{i}\right)^{2}\right] .
\end{aligned}
$$

Denoting the function in brackets by $H_{N-1}$, we see that

$$
\begin{aligned}
& \left(\partial F / \partial \theta^{N-1}\right)\left(\theta^{1}, \ldots, \theta^{N-1}\right)=0 \text { if and only if } \\
& \theta^{N-1}=\theta_{0}^{N-1}=\pi / 4 \text { or } H_{N-1}\left(\theta^{1}, \ldots, \theta^{N-1}\right)=0 .
\end{aligned}
$$

We now show that there is a $\beta>p+1$ such that $H_{N-1}$ does not vanish whenever $p$ satisfies (*). By applying 3.1(2) and 3.1(3) we find 


$$
\begin{aligned}
H_{N-1}= & (\beta-p)\left[\left(\left(\frac{N p+1}{\beta}\right)^{2}-N^{2}\right) C(1, N-2)^{2} S(1, N-2)^{2}\right. \\
& \left.+\sum_{i=1}^{N-2} S(i, N-2)^{2} C(1, i-1)^{2} C(i+1, N-2)^{2}\right] \\
& \cdot \sin ^{2} \theta^{N-1} \cos ^{2} \theta^{N-1}+(\beta-p-1) C(1, N-2)^{2} \\
= & A_{N-1} \sin ^{2} \theta^{N-1} \cos ^{2} \theta^{N-1}+D_{N-1} .
\end{aligned}
$$

According to 3.2 the only possible minima for this function of $\theta^{N-1}$ occur at $0, \pi / 2$ or $\pi / 4$. Since $H_{N-1}>0$ at $\left(\theta^{1}, \ldots, \theta^{N-2}, 0\right)$ and $\left(\theta^{1}, \ldots, \theta^{N-2}, \pi / 2\right)$ we may assume $H_{N-1}$ attains its minimum when $\theta^{N-1}$ $=\pi / 4$. Hence

$$
\begin{aligned}
4 S(1, N- & 2)^{-2} C(1, N-2)^{-2}(\beta-p)^{-1} H_{N-1} \\
> & \left(\frac{N p+1}{\beta}\right)^{2}-N^{2}+\sum_{i=1}^{N-2} S(1, i-1)^{-2} \cos ^{-2} \theta^{i} \\
& \quad+4\left(1-(\beta-p)^{-1}\right) S(1, N-2)^{-2} \\
> & ((N p+1) / \beta)^{2}-N^{2}+\left(N-2+2\left(1-(\beta-p)^{-1}\right)^{1 / 2}\right)^{2}
\end{aligned}
$$

by 3.3. Since $\beta>p+1$ this last expression is greater than $((N p+1) / \beta)^{2}-$ $4 N+4$. Now note that

$$
((N p+1) / \beta)^{2}-4 N+4>0 \text { if } \beta<(N p+1) / 2(N-1)^{1 / 2}
$$

and that there exists $\beta$ such that

$$
(N p+1) / 2(N-1)^{1 / 2}>\beta>p+1
$$

whenever $p>\left(2(N-1)^{1 / 2}-1\right) /\left(N-2(N-1)^{1 / 2}\right)$.

Assume inductively that

$$
\partial F / \partial \theta^{j}=0 \text { only when } \theta^{j}=\theta_{0}^{j}, \quad k<j<N-1 .
$$

Then by applying (4), 3.1(1)-3.1(3) again, we obtain

$$
\begin{aligned}
& \left(\partial F / \partial \theta^{k}\right)\left(\theta^{1}, \ldots, \theta^{k}, \theta_{0}^{k+1}, \ldots, \theta_{0}^{N-1}\right) \\
& \quad=-2 \beta^{2}(N p+1)^{-2}(\beta-p) m_{0}^{2 \beta-2 p} h_{k}^{2 \beta-2 p}\left((N-k) \cot \theta^{k}-\tan \theta^{k}\right) H_{k},
\end{aligned}
$$

where $h_{k}\left(\theta^{1}, \ldots, \theta^{k}\right)=h\left(\theta^{1}, \ldots, \theta^{k}, \theta_{0}^{k+1}, \ldots, \theta_{0}^{N-1}\right)$ and

$$
\begin{aligned}
H_{k}= & \left(\frac{N p+1}{\beta}\right)^{2}-N^{2}+\sum_{i=1}^{k-1} S(1, i-1)^{-2} \cos ^{-2} \theta^{i} \\
& +\left(1-(\beta-p)^{-1}\right) S(1, k-1)^{-2} \cos ^{-2} \theta^{k} \\
& +\left((N-k)^{2}-(N-k)(\beta-p)^{-1}\right) S(1, k)^{-2}
\end{aligned}
$$


From 3.3 and $\beta>p+1$ we infer

$$
\begin{aligned}
H_{k} \geqslant & ((N p+1) / \beta)^{2}-N^{2} \\
& +\left(k-1+\left(1-(\beta-p)^{-1}\right)^{1 / 2}+\left((N-k)^{2}-(N-k)(\beta-p)^{-1}\right)^{1 / 2}\right)^{2} \\
& >((N p+1) / \beta)^{2}-N^{2}+\left(k-1+\left((N-k)^{2}-(N-k)\right)^{1 / 2}\right)^{2} .
\end{aligned}
$$

Observe $(N-k)^{2}-(N-k) \geqslant(N-k-1)^{2}$, so that

$$
H_{k}>((N p+1) / \beta)^{2}-4 N+4>0 \text {. }
$$

By induction we conclude that whenever $p$ satisfies (*) there is a $\beta$ such that

$$
d F\left(\theta^{1}, \ldots, \theta^{N-1}\right)=0 \Leftrightarrow \theta^{i}=\theta_{0}^{i}, \quad i=1, \ldots, N-1 .
$$

Thus (II) is proved.

Consider the special case $N=3$. From (5) we have

$$
\begin{aligned}
H_{2}\left(\theta^{1}, \pi / 4\right)= & \frac{1}{4}(\beta-p)\left[\left(((3 p+1) / \beta)^{2}-9\right) \cos ^{2} \theta^{1} \sin ^{2} \theta^{1}+\sin ^{2} \theta^{1}\right] \\
& +(\beta-p-1) \cos ^{2} \theta^{1} .
\end{aligned}
$$

If $\alpha=\beta-p$, then

$$
\begin{aligned}
4 H_{2}\left(\theta^{1}, \pi / 4\right)= & \alpha\left[((3 p+1) /(\alpha+p))^{2}-9\right] \cos ^{2} \theta^{1} \sin ^{2} \theta^{1} \\
& +\alpha \sin ^{2} \theta^{1}+4(\alpha-1) \cos ^{2} \theta^{1} .
\end{aligned}
$$

By 3.2 either $\mathrm{H}_{2}>\mathrm{O}$ or $4 \mathrm{H}_{2}$ attains a minimum value of

$$
\begin{aligned}
& \left(4 \alpha\left[((3 p+1) /(\alpha+p))^{2}-9\right]\right)^{-1} \\
& \quad \cdot\left\{\left(\alpha\left[((3 p+1) /(\alpha+p))^{2}-9\right]+\alpha+4(\alpha-1)\right)^{2}-16 \alpha(\alpha-1)\right\} .
\end{aligned}
$$

For $\alpha=(p+1) / 2$ the expression in the braces becomes $-16\left(\alpha^{2}-\alpha-1\right)$, which is negative for $p>3$. Thus $H_{2}>0$ for $p>3$.

Now compute

$$
\begin{aligned}
\left(\partial F / \partial \theta^{1}\right)\left(\theta^{1}, \pi / 4\right) & \\
= & -2 \beta^{2}(3 p+1)^{-2} m_{0}^{2 \beta-2 p} h_{1}^{2 \beta-2 p}\left(2 \cot \theta^{1}-\tan \theta^{1}\right) \\
& \cdot\left[(\beta-p)\left(((3 p+1) / \beta)^{2}-9\right)+(4(\beta-p)-2) \sin ^{-2} \theta^{1}\right. \\
& \left.+(\beta-p-1) \cos ^{-2} \theta^{1}\right] .
\end{aligned}
$$

If we apply 3.3 and use $\alpha=(p+1) / 2$, then the minimum value of the expression in the brackets is 


$$
\begin{gathered}
\alpha\left(((3 p+1) / \beta)^{2}-9\right)+\left((4 \alpha-2)^{1 / 2}+(\alpha-1)^{1 / 2}\right)^{2} \\
=2(\alpha-1)^{1 / 2}(4 \alpha-2)^{1 / 2}-3,
\end{gathered}
$$

which is positive whenever $p \geqslant 3$.

3.5. CoROLlaRY. If $N$ and $p$ satisfy the hypotheses of 3.4 and

is defined by

$$
\Psi: U \times \bigwedge_{1} \mathbf{R}^{N} \rightarrow \mathbf{R}
$$

$$
\Psi(u, \alpha)=\mathcal{H}^{N p}\left(\pi^{-1}\{u\}\right)|\alpha|,
$$

then $L_{0}=\mathcal{I C}^{1}\left\llcorner(L \cap U) \wedge \xi_{1} \in \mathrm{I}_{1}^{\mathrm{loc}}(U)\right.$ is absolutely $\Psi$ minimizing, where $\xi_{1}(u)$ $=u /|u|$.

Proof. Let $K$ be a compact subset of $U$ and $\varphi$ be as in 3.4. From 3.4(2)

$$
L_{0} L K(\varphi)=\int_{L_{0} L K} \Psi .
$$

Let $Q \in \mathbf{I}_{1}(U)$ be such that $Q-L_{0} L K \in \mathscr{I}_{1}(U)$. Since $\mathscr{I}_{1}(U)=\mathscr{B}_{1}(U)$, 3.4(1) implies $Q(\varphi)=L_{0} L K(\varphi)$. Use 3.4(3) to infer $\int_{Q} \Psi>Q(\varphi)$. Thus

$$
\int_{L_{0} L K} \Psi=L_{0} L K(\varphi)=Q(\varphi)<\int_{Q} \Psi .
$$

3.6. REMARK. The current $L_{0}$ is $\Psi$ minimizing with respect to chains with real coefficients. To see this take $Q \in \mathrm{F}_{1}(U)$ such that $\mathbf{M}(Q)<\infty$ and $Q-L_{0} L K \in \mathbf{Z}_{1}(U)$ and use the above argument to conclude

$$
\int_{L_{0} L K} \Psi=\inf \left\{\int_{Q} \Psi: Q-L_{0} L K \in \mathbf{Z}_{1}(U), \mathbf{M}(Q)<\infty\right\} .
$$

4. The examples.

4.1. Notation. Let $r$ denote the radial coordinate function in $\mathbf{R}^{p+1}-\{0\}$. Define $\Upsilon_{0}, \Upsilon \in \mathcal{E}^{p}\left(\mathbf{R}^{p+1}-\{0\}\right)$ by

$$
\Upsilon_{0}=* d r \text { and } \Upsilon(y)=((p+1) \alpha(p+1))^{-1}|y|^{-p} \Upsilon_{0}(y) .
$$

Denote the $p$-vectorfield dual to $\Upsilon_{0}$ by $Y_{0}$ and note that $Y_{0}(y)$ spans the tangent space of the sphere of radius $|y|$ at $y$. Also

$$
\|\Upsilon(y)\|=((p+1) \alpha(p+1))^{-1}|y|^{-p} \text { and } d \Upsilon=0 .
$$

Defining $\pi: \mathbf{R}^{N(p+1)} \rightarrow \mathbf{R}^{N}$ and $U$ as in $\S 1$, we denote $X=\pi^{-1}(U)$ and $S=\mathbf{R}^{N(p+1)}-X$ and define

$$
V(x)= \begin{cases}V_{p} \circ \pi(x) & \text { if } x \in X, \\ 0 & \text { if } x \in S .\end{cases}
$$

Identify $\mathbf{R}^{N(p+1)}$ with $\left(\mathbf{R}^{p+1}\right)^{N}$ and let $\pi_{j}: \mathbf{R}^{N(p+1)} \rightarrow \mathbf{R}^{p+1}$ be the projection 
onto the $j$ th factor. Define

$$
\Omega=\pi_{i}^{\#} \wedge \wedge \cdots \wedge \pi_{N}^{\#} \Upsilon \in \mathcal{E}^{N p}(X) .
$$

Identifying

$$
\bigwedge_{N p} \mathbf{T}_{x} \mathbf{R}^{N(p+1)} \text { with } \underset{\Sigma_{i} k_{i}=N p}{\bigoplus} \bigotimes_{i=1}^{N}\left(\widehat{k}_{i} \mathbf{T}_{\pi_{i}(x)} \mathbf{R}^{p+1}\right)
$$

we define the $N p$ vectorfield $Y$ on $X$ by

$$
Y(x)=Y_{0}\left(\pi_{1}(x)\right) \otimes \cdots \otimes Y_{0}\left(\pi_{N}(x)\right)
$$

Since $Y_{0}$ is dual to $\Upsilon_{0}$, it is easy to see that $Y$ is dual to $\Omega_{0}=|\Omega|^{-1} \Omega$. Finally, note that $d \Omega=0$ and

$$
\begin{aligned}
|\Omega(x)| & =[(p+1) \alpha(p+1)]^{-N}\left|\pi_{1}(x)\right|^{-p} \cdots\left|\pi_{N}(x)\right|^{-p} \\
& =\left(V_{p} \circ \pi(x)\right)^{-1}=V(x)^{-1} .
\end{aligned}
$$

4.2. Definition. By the oriented cone through $\left(S^{p}\right)^{N} \subset \mathbf{R}^{N(p+1)}$, we mean the locally integral current $C \in \mathrm{I}_{N p+1}^{\text {loc }}\left(\mathbf{R}^{N(p+1)}\right)$ defined by the formula

$$
C=\mathcal{F}^{N p+1} L \pi^{-1}(L) \wedge \xi \wedge Y,
$$

where $\xi$ is the vectorfield $\xi(x)=x /|x|$.

4.3. Theorem. If $N=3$ and $p>3, N=4$ and $p>5$, or $N>5$ and $p>N-1$, then $C$ is absolutely area minimizing. Moreover, for any compact set $K \subset \mathbf{R}^{N(p+1)}, C L K$ is the unique area minimizing integral current with boundary $\partial(C L K)$.

Proof. Note that if $N$ and $p$ satisfy any of the above conditions then they satisfy the hypotheses of 3.4 and $p>N-2$.

Let $\varphi$ be as in 3.4 and define $\varphi_{0} \in \mathcal{E}^{N p+1}(X)$ by the formula

Let $K$ be a compact subset of $\mathbf{R}^{N(p+1)}$.

$$
\varphi_{0}=\pi^{\sharp} \varphi \wedge \Omega \text {. }
$$

From 3.4(2) we have

$$
\begin{aligned}
C L K\left(\varphi_{0}\right) & =\int_{K \cap \pi^{-1}(L)}\left\langle\xi \wedge Y, \pi^{\sharp} \varphi \wedge \Omega\right\rangle d \mathcal{F}^{N p+1} \\
& =\int_{K \cap \pi^{-1}(L)}\left\langle\xi, \pi^{\sharp} \varphi\right\rangle V^{-1} d \mathcal{F}^{N p+1} \\
& =\int_{K \cap \pi^{-1}(L)}\langle\pi(x) /|x|, \varphi(\pi(x))\rangle V(x)^{-1} d \mathcal{F}^{N p+1}(x) \\
& =\int_{K \cap \pi^{-1}(L)} V_{p}(\pi(x)) V(x)^{-1} d \mathcal{F}^{N p+1}(x) \\
& =\mathcal{I C}^{N p+1}\left(K \cap \pi^{-1}(L)\right)=\mathbf{M}(C L K) .
\end{aligned}
$$


Since $\mathcal{F}^{N(p+1)-1}(S)=0, \varphi_{0}$ satisfies [F3, 6.2(I), (II)]. Applying 4.1, 3.4(3) and 3.4(1) we obtain

$$
\begin{gathered}
\left\|\varphi_{0}\right\|<\left\|\pi^{\sharp} \varphi\right\|\|\Omega\|=(\|\varphi\| \circ \pi) V^{-1}<\left(V_{p} \circ \pi\right) V^{-1}=1, \\
d \varphi_{0}=\pi^{\sharp} d \varphi \wedge \Omega-\pi^{\sharp} \varphi \wedge d \Omega=0 .
\end{gathered}
$$

Thus [F3, 6.2(III)] is satisfied and we conclude that there exists $\alpha \in$ $\mathbf{F}^{N p+1}\left(\mathbf{R}^{N(p+1)}\right)$ associated with $\varphi_{0}$.

Let $Q \in \mathbf{I}_{N p+1}\left(\mathbf{R}^{N(p+1)}\right)$ be such that

$$
Q-C L K \in \mathscr{Z}_{N p+1}\left(\mathbf{R}^{N(p+1)}\right)=\mathscr{B}_{N p+1}\left(\mathbf{R}^{N(p+1)}\right) .
$$

Since $d \varphi_{0}=0$ we use [F3, 4.6(2)] to infer $d \alpha=0$ and conclude $\alpha(Q-C L K)$ $=0$. Whenever $p>N-2$ the dimension of $S$.is less than $N p+1$ and we see that $\mathcal{T}^{N p+1}(S)=0$. Thus the form $\varphi_{0}$ is continuous $\|Q\|$ almost everywhere so that

$$
\alpha(Q)=\int\left\langle\vec{Q}, \varphi_{0}\right\rangle d\|Q\|
$$

by [F3, p. 378]. Because $\left\|\varphi_{0}\right\|<1$,

$$
\mathbf{M}(Q) \geqslant \int\left\langle\vec{Q}, \varphi_{0}\right\rangle d\|Q\|=\alpha(Q)=\alpha(C L K)=\mathbf{M}(C L K) .
$$

To prove uniqueness observe that equality holds above if and only if spt $Q \subset \pi^{-1}(L)=\operatorname{spt} C$. Since $\mathcal{T}^{N p+1}(S)=0$ it will suffice to prove $(Q-$ $C L K) \mid \mathscr{D}^{N p+1}(X)=0$. Use $[\mathrm{F3}, 4.1 .31]$ and the fact that $B=X \cap$ spt $C$ is a connected $(N p+1)$-dimensional manifold to find $s \in \mathbf{R}$ such that

$$
(Q-C L K)-s\left(\mathcal{F}^{N p+1}\llcorner B) \wedge \xi \wedge Y=0 .\right.
$$

However, $\operatorname{spt}(Q-C L K)$ is bounded while

$$
\operatorname{spt}\left(\left(\Im^{\mathcal{C}_{p}+1}\llcorner B) \wedge \xi \wedge Y\right)=B\right.
$$

is not. Hence $s=0$.

4.4. REMARK. The cone $C$ also minimizes area with respect to chains with real coefficients. To prove this fact the only change required in the proof of 4.3 is to take $Q \in \mathbf{F}_{N p+1}\left(\mathbf{R}^{N(p+1)}\right)$ such that $\mathbf{M}(Q)<\infty$ and $Q-C L K \in$ $\mathbf{Z}_{N p+1}\left(\mathbf{R}^{N(p+1)}\right)$.

\section{REFERENCES}

[B] D. E. Bindschadler, Invariant and singular solutions to the Plateau problem in Riemannian manifolds, Ph.D. Thesis, Indiana Univ., 1976.

[BGG] E. Bombieri, E. DeGiorgi and E. Guisti, Minimal cones and the Bernstein problem, Invent. Math. 7 (1969), 243-268.

[F1] H. Federer, Some theorems on integral currents, Trans. Amer. Math. Soc. 117 (1965), 43-67.

[F2]

[F3] Geometric measure theory, Springer-Verlag, New York, 1969. $351-407$. , Real flat chains, cochains and variational problems, Indiana J. Math. 24 (1974), 
[FF] H. Federer and W. H. Fleming, Normal and integral currents, Ann. of Math. (2) 72 (1960), 458-520.

[L] H. B. Lawson, The equivariant Plateau problem and interior regularity, Trans. Amer. Math. Soc. 173 (1973), 231-249.

[S] Plinio Simoes, On a class of minimal cones in $\mathbf{R}^{n}$, Bull. Amer. Math. Soc. 80 (1974), 488-489.

Department of Mathematics, Wayne State Universtty, Detrott, Michoan 48202 